\title{
Effect of Operating Parameters of an Electromagnetic Refining Process on the Solidified Structure
}

\author{
Kento SUGIURA and Kazuhiko IWAI ${ }^{11}$ \\ Graduate Student of Nagoya University, Furo-cho, Chikusa-ku, Nagoya 464-8603 Japan. \\ 1) Department of Materials Processing Engineering, Graduate School of Engineering, Nagoya University, Furo-cho, Chikusa- \\ ku, Nagoya 464-8603 Japan.
}

(Received on February 27, 2004; accepted in final form on May 6, 2004)

\begin{abstract}
Effect of operating parameters of a refining process on the solidified structure such as frequency and intensity of an alternating current has been experimentally examined by use of $\mathrm{Sn}-10 \mathrm{mass} \% \mathrm{~Pb}$ alloy. In the refining process of a solidified structure in this manuscript, a static magnetic field and an alternating electrical current are simultaneously imposed on the local area of a metal or an alloy using copper electrodes. Concerning the intensity of the electrical current, threshold exists for the refining of the solidified structure, and the refined structure is mainly observed around the electrode in the case that the magnitude of the electrical current is around the threshold value. Imposition of the electromagnetic force in the initial stage of the solidification is crucial to obtain the refined structure in this process and the imposition in the middle stage has no refining function.
\end{abstract}

KEY WORDS: solidification; crystal refinement; electromagnetic processing of materials; local imposition of oscillation.

\section{Introduction}

Mechanical properties of materials such as strength, elongation and toughness highly depend upon their grain sizes, which are almost determined in a solidification process. Then the control of their solidified structure is essential for products with good mechanical properties. Heat flow control during solidification is an ordinary method to control grain sizes of solidified structures though it is difficult to apply this method to casting of large size products. Though inoculation can also refine solidified structures, it is sometimes harmful for recycling of products because of added elements.

Compression waves are attractive tools to refine solidified structures. ${ }^{1)}$ However, non-contacting introduction of the compression waves ${ }^{2,3)}$ is strongly desired for metals and alloys with high temperature melting point to avoid the difficulties such as dissolution or destruction of a transmitter.

Direct excitation of electromagnetic vibrations in a solidifying metal is another method for refining of solidified structures. Vives ${ }^{4)}$ and Radjai et al. ${ }^{5,6)}$ refined aluminum alloy structures by applying the vibration, which was excited by the simultaneous imposition of a static magnetic field and an alternating current on the whole of the alloys during solidification.

On the other hand, the authors proposed the other refining method in which a static magnetic field and an alternating current are simultaneously imposed on a local volume of a metal or an alloy. ${ }^{7,8)}$ This method is useful for a large size product because of local imposition of the vibration.
In this paper, the effect of operating parameters in the refining method proposed by the authors ${ }^{7)}$ on the solidified structure has been experimentally examined.

\section{Experiment}

\subsection{Experimental Apparatus and Procedure}

A sample of a $\mathrm{Sn}-10 \mathrm{mass} \% \mathrm{~Pb}$ alloy was prepared by alloying a pure molten $\mathrm{Sn}(99.9 \%)$ and a pure molten $\mathrm{Pb}$ $(99.9 \%)$. Its liquidus and eutectic temperatures are $219^{\circ} \mathrm{C}$ and $183^{\circ} \mathrm{C}$, respectively as shown in the phase diagram of $\mathrm{Sn}-\mathrm{Pb}$ alloy (Fig. 1). A schematic view of the experimental apparatus is shown in Fig. 2. A rectangular glass vessel of length $40 \mathrm{~mm}$, width $25 \mathrm{~mm}$ was filled with a liquid sample

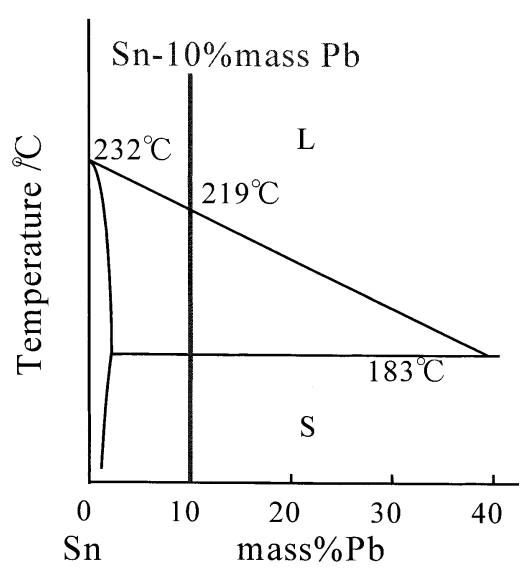

Fig. 1. The phase diagram of $\mathrm{Sn}-\mathrm{Pb}$ alloy. 


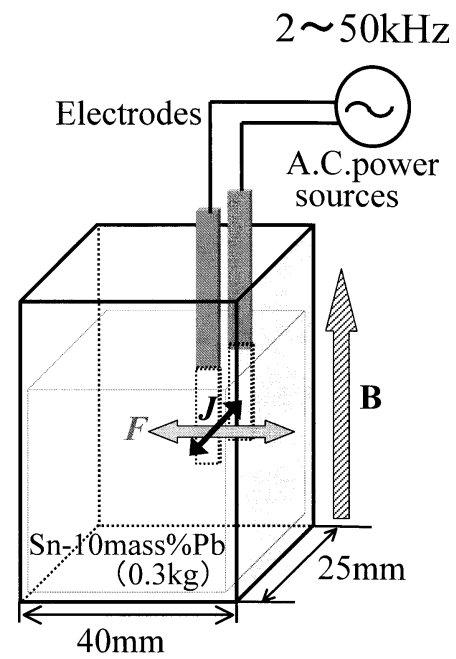

Fig. 2. Schematic view of experimental apparatus.

of $0.3 \mathrm{~kg}$ (about $40 \mathrm{~mm}$ depth) and was set in the bore of a supper-conducting magnet. This magnet imposed an upward vertical static magnetic field of $10 \mathrm{~T}$ on the sample. An alternating current of $2 \mathrm{kHz}$ was supplied to the sample in the particular period depending on the experimental condition through a couple of copper electrodes (thickness $2 \mathrm{~mm} \times$ width $5 \mathrm{~mm}$ ) inserted into the sample in the vicinity of a short wall of the vessel. Furthermore, the super-conducting magnet, the sample and the electrodes were separately supported to prevent the propagation of vibration to the sample. After the solidification of the sample, its vertical or horizontal cross-section cut parallel to the long wall was observed.

\subsection{Effect of Electromagnetic Vibration on the Solidi- fied Structure}

Imposition of the electrical current started when the sample temperature became $250^{\circ} \mathrm{C}$ which is $31^{\circ} \mathrm{C}$ above the liquidus temperature, and it finished when the sample temperature became $170^{\circ} \mathrm{C}$, which is $13^{\circ} \mathrm{C}$ below the eutectic temperature, while the magnetic field was imposed on the sample during the whole period of the solidification. Intensity of the static magnetic field and effective value of the alternating current adopted in the experiment are $10 \mathrm{~T}$ and $90 \mathrm{~A}$, respectively.

The macrostructures of the horizontal cross-section with and without the electrical current are shown in Fig. 3 . When the static magnetic field and the alternating current were simultaneously imposed on the sample, an electromagnetic vibration was excited in it. In this case, the solidified structure was refined in the whole region of the sample, while in the case without the electromagnetic vibration (without electrical current) coarse grains are observed in the whole region. That is, the electromagnetic vibration excited in the local region can refine the structure.

Temperature profiles in the samples with and without the vibration were measured at three positions. These were $20 \mathrm{~mm}$ above from the vessel bottom and along the long wall. The results are shown in Fig. 4. The temperature difference in the sample was less than a few Kelvins before the solidification in the samples with and without the vibration. Recalescence was clearly observed in the order of the positions $\mathrm{B}, \mathrm{A}$ and $\mathrm{C}$ in the sample without the vibration.

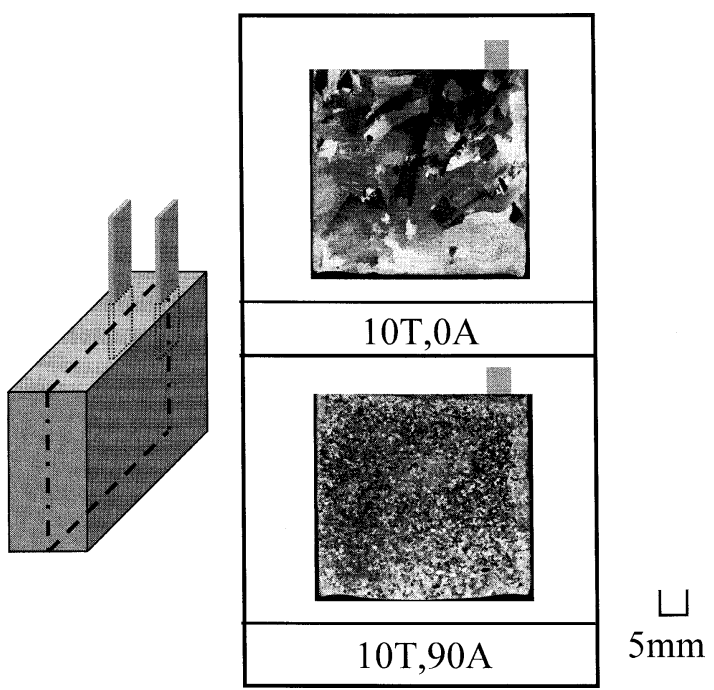

Fig. 3. Macrostructures of the vertical cross-section with and without electrical current.
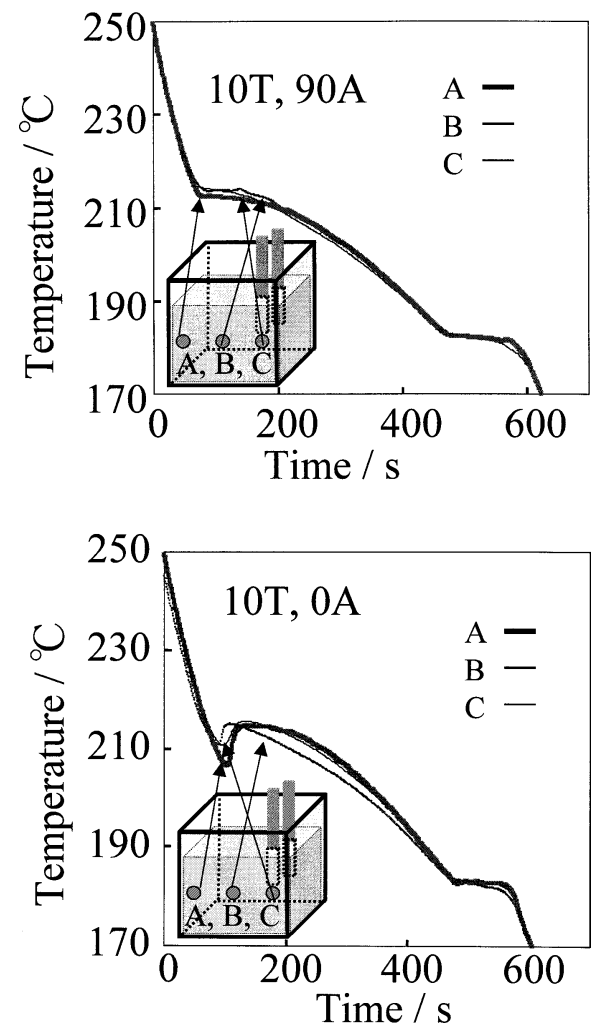

Fig. 4. Temperature profiles with and without vibration

On the other hand, it was not clearly observed in the sample with the vibration. That is, the electromagnetic vibration might affect nucleation in this experiment. After the recalescence, the temperature at the position B was lower than those at the other two positions in the sample without the vibration while the temperature was almost uniform in the sample with the vibration.

To confirm whether the refinement of the structure is caused or not by the electromagnetic vibration in the alloy, the electrodes shown in Fig. 5 were used, in which the lower part of the electrodes was electrically insulated and a copper block was inserted between them just above the insulated part. When these electrodes were used in the experiment, a vibrating Lorentz force was not excited in the sam- 

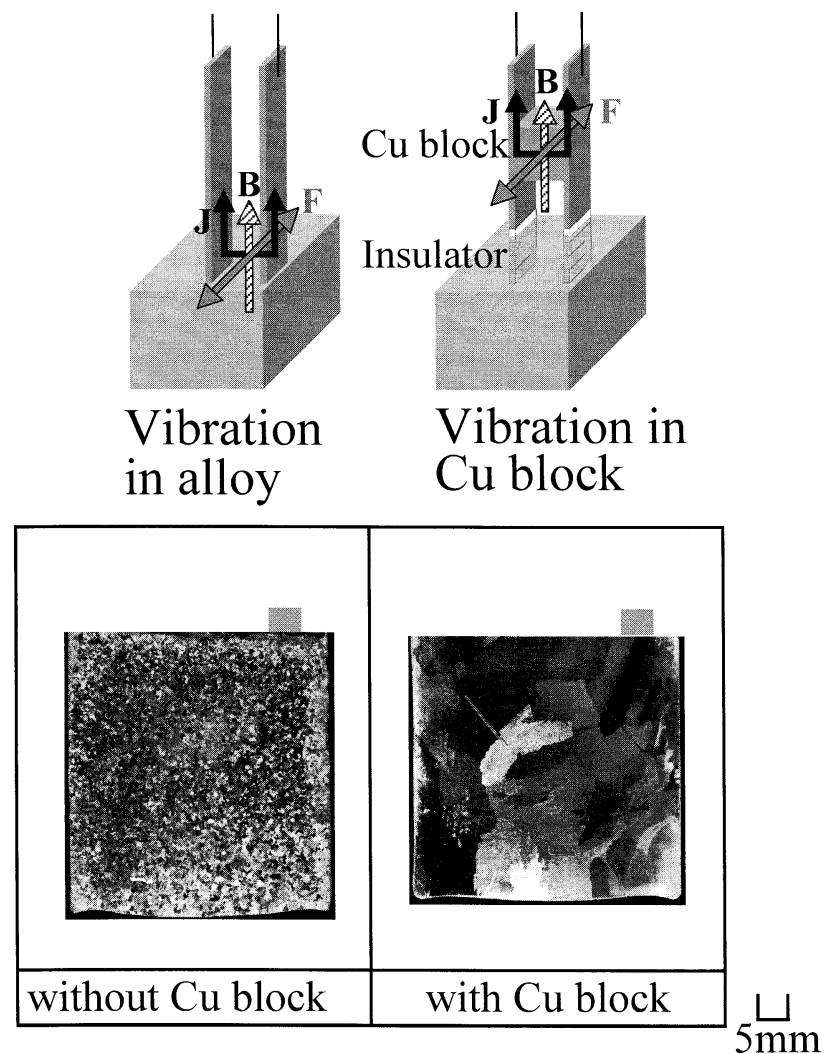

Fig. 5. Macrostructures using electrodes with and without $\mathrm{Cu}$ block. ple but was excited in the copper block and it might propagate to the sample through the electrodes. Since the macrostructure of the sample solidified with the copper inserted electrodes is coarse as shown in Fig. 5, the electrical current flow in an alloy is crucial for refinement of a solidified structure in this process.

\subsection{Effect of Frequency}

Theoretical distributions of pressure change, $p$ and velocity, $v_{x}$ in a sample accompanying compression waves excited by the simultaneous imposition of a static magnetic field and an alternating current are derived ${ }^{7)}$ as:

$$
\begin{aligned}
v_{x}= & V_{\mathrm{m}} \frac{N \gamma}{\sqrt{1+N^{2} \gamma^{4}}} \\
& \times\left[\frac{1}{\gamma \sqrt{N}} \exp \left(-\frac{x}{\delta}\right) \sin \left(-\frac{x}{\delta}+\omega t+\frac{\pi}{4}-\phi\right)\right. \\
& \left.+\frac{\cos (k x-k l)}{\sin (k l)} \cos (\omega t-\phi)\right] \\
p= & P_{\mathrm{m}} \frac{N}{\sqrt{1+N^{2} \gamma^{4}}} \times\left[\exp \left(-\frac{x}{\delta}\right) \sin \left(-\frac{x}{\delta}+\omega t-\phi\right)\right. \\
& \left.+\frac{\sin (k x-k l)}{\sin (k l)} \sin (\omega t-\phi)\right]
\end{aligned}
$$

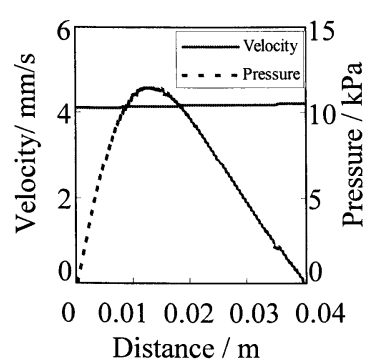

$\underline{\mathbf{2 k H z}}$

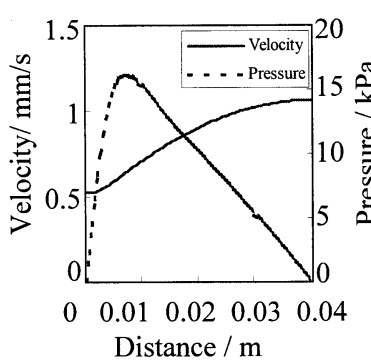

$\underline{10 \mathrm{kHz}}$

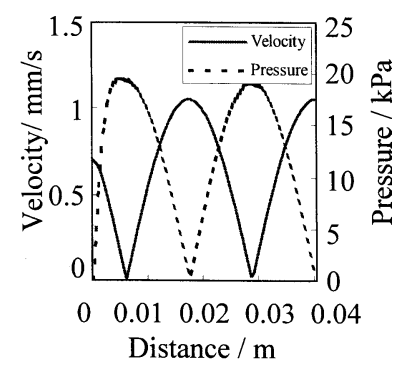

$\underline{50 \mathrm{kHz}}$

(a)

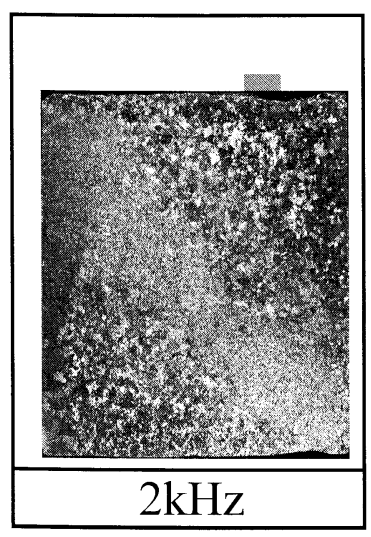

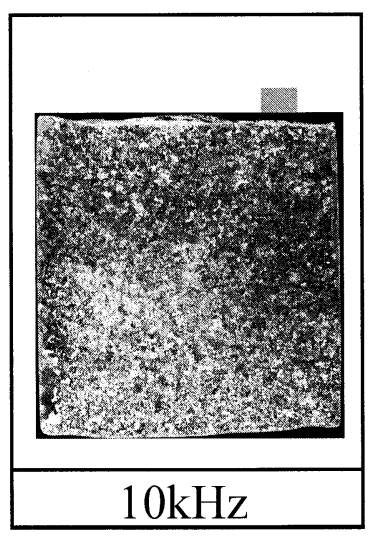

(b)
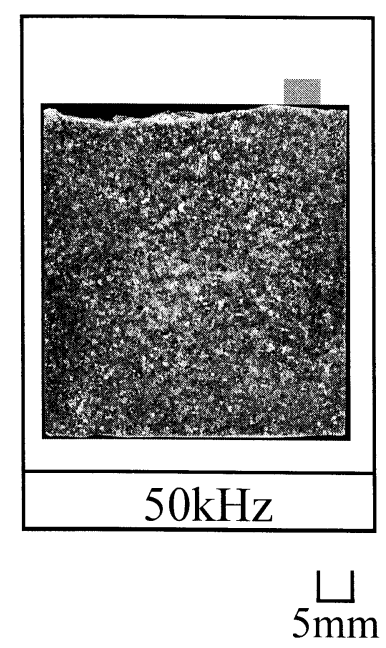

Fig. 6. Effect of frequency on the structure. (a) Theoretical distributions of pressure and velocity, (b) macrostructure. 
where

$$
\begin{aligned}
a^{2} & \equiv c^{2}+\frac{B_{\mathrm{DC}}^{2}}{\rho_{0} \mu}, \quad k \equiv \frac{\omega}{a}, \quad N \equiv \frac{2 c^{2}}{\delta^{2} \omega^{2}}, \\
P_{\mathrm{m}} & \equiv \delta B_{\mathrm{DC}} J_{\mathrm{AC}}, \quad V_{\mathrm{m}} \equiv \frac{\delta B_{\mathrm{DC}} J_{\mathrm{AC}}}{\rho_{0} c}, \quad \gamma \equiv \frac{a}{c}, \\
\delta & \equiv \sqrt{\frac{2}{\mu \sigma \omega}} \quad \text { and } \quad \phi \equiv \tan ^{-1}\left(N \gamma^{2}\right)+\frac{\pi}{4}
\end{aligned}
$$

The symbols in these equations are summarized in the Nomenclature. The pressure change and velocity in the alloy under the experimental conditions mentioned below were calculated from the above equations and are shown in Fig. 6(a). The distributions of the pressure change and velocity depend on the frequency of the electrical current.

The effect of the frequency on the structure was examined in the experiment. The constant magnetic field of $10 \mathrm{~T}$ and the constant current intensity of $50 \mathrm{~A}$ with different frequencies of 2, 10 and $50 \mathrm{kHz}$ were adopted in this experiment. The obtained macrostructures are shown in Fig. 6(b). All of the samples are refined though the theoretical distributions of the pressure change and velocity in the samples highly depend on the frequency. That is, the frequency of the alternating current does not affect the structure in this process. Compression waves are physical phenomena in which fluid motion and pressure change simultaneously propagate. If one of them has a refining function in this process, a refined structure should be observed at its loops and a coarse structure should be observed at its nodes. However, the obtained structures are almost uniformly refined. Therefore, both the fluid motion and the pressure change accompanying the compression waves might have the refining function in this process, or the other refining mechanism might exist.

\subsection{Effect of Electrical Current Magnitude}

The samples were solidified under the different magnitude of the alternating electrical current with $2 \mathrm{kHz}$ frequency while the intensity of the static magnetic field was fixed at 10 T. The macrostructures are shown in Fig. 7. The structures are not refined if the current magnitude is equal to or less than $5 \mathrm{~A}$ while it is refined when the magnitude is $50 \mathrm{~A}$ and $90 \mathrm{~A}$. The refining effect is observed only in the vicinity of the electrode in the case of $20 \mathrm{~A}$. That is, threshold of the electrical current intensity for refinement must be exist around $20 \mathrm{~A}$ in this experimental condition.

The intersection numbers between grain boundaries and each diagonal of a $10 \mathrm{~mm} \cdot 10 \mathrm{~mm}$ square were measured for the quantitative evaluation of the refinement. The positions of the four squares and the evaluated intersection numbers are shown in Fig. 8 as the function of the magnitude of the electrical current. The intersection number increases at the all positions as the magnitude of the electrical current increases. Refining effect is remarkable at the position $\mathrm{C}$ which is the nearest the electrodes. In the case of $50 \mathrm{~A}$, the refinement is achieved except the position B. This means that the refining effect decreases as the increase of distance from the electrode.

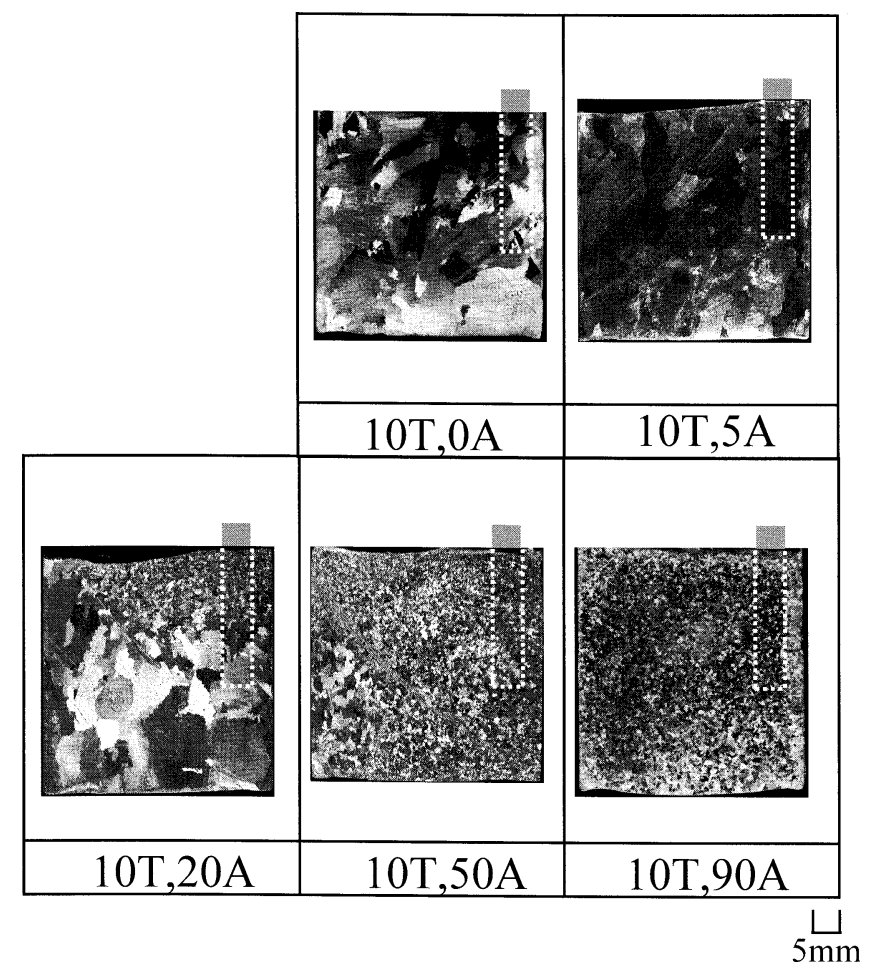

Fig. 7. Macrostructures under the different magnitude of the electrical current.

\section{Electrode}
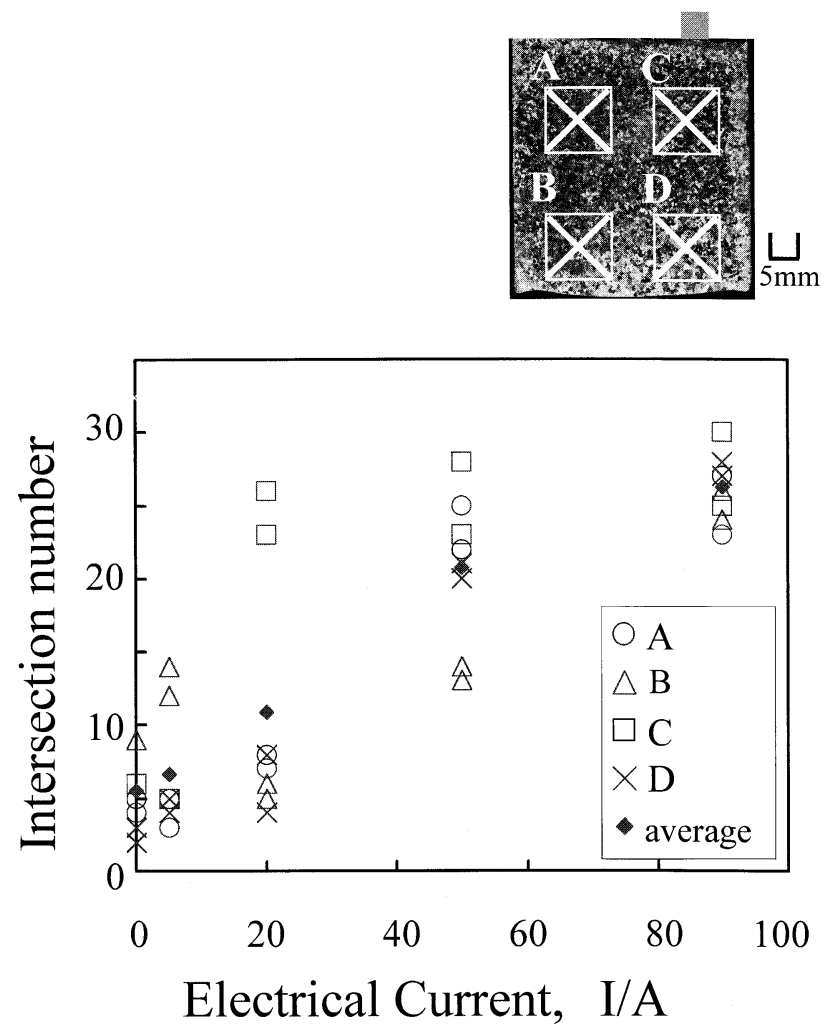

Fig. 8. Intersection number between grain boundary and diagonal of $10 \mathrm{~mm} \times 10 \mathrm{~mm}$ square as a function of electrical current magnitude.

\subsection{Effect of Imposing Duration of Electrical Current on Solidified Structure}

The imposing durations of the alternating current of $90 \mathrm{~A}$ 


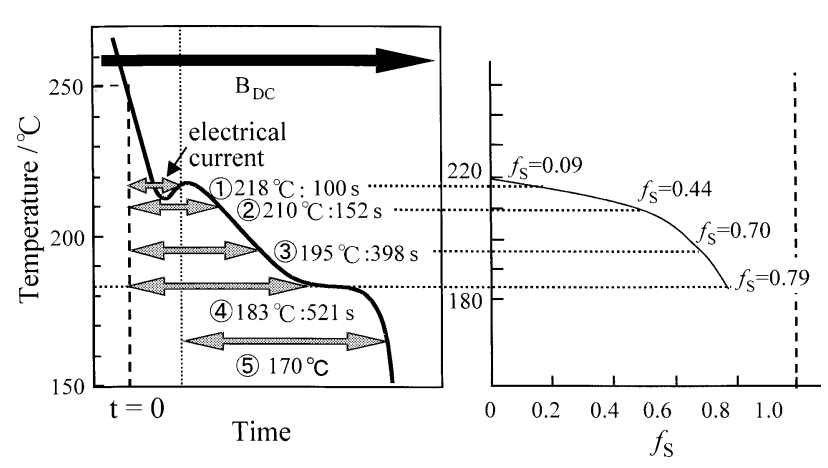

Fig. 9. Imposing duration of electrical current and corresponding solid fraction.

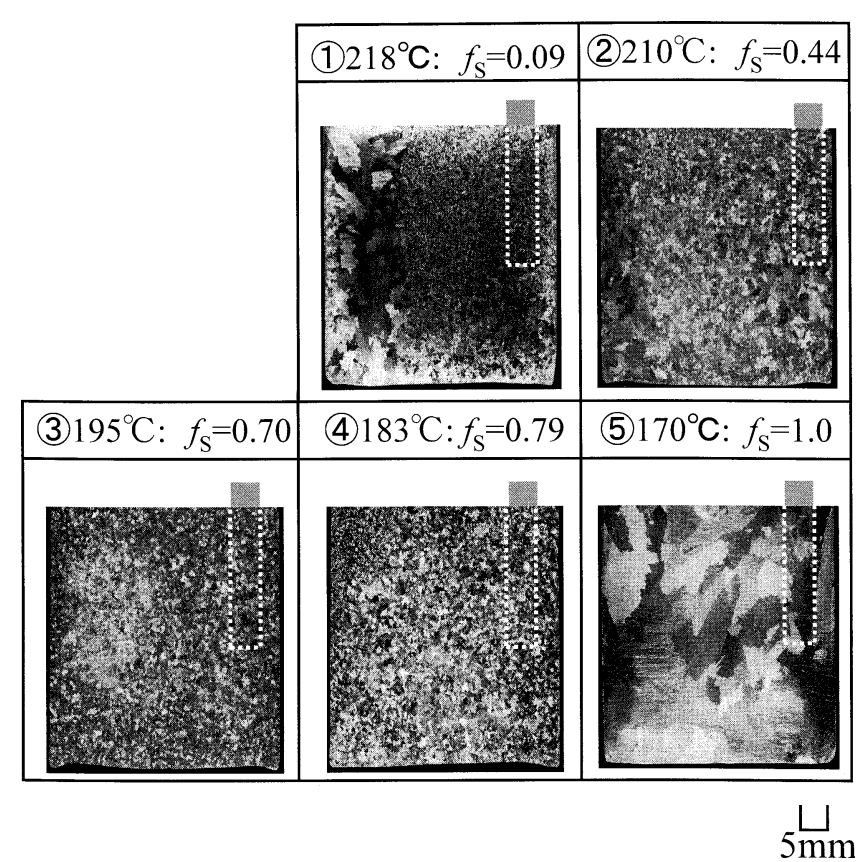

Fig. 10. Effect of imposing duration of electrical current on macrostructure.

adopted in this section and corresponding mass solid fractions when the electrical current was turned off are shown in Fig. 9. For the samples 1, 2, 3 and 4, the electrical current was turned on when the sample temperature became $250^{\circ} \mathrm{C}$ at which the alloy was liquid. For the sample 1, it was turned off when the recovery of the temperature accompanying recalescence finished, while it was turned off when the temperature reached at 210,195 and $183^{\circ} \mathrm{C}$ for the samples 2, 3 and 4, respectively. For the sample 5, the current was turned on when the temperature recovered and it was turned off when the temperature decreased to $170^{\circ} \mathrm{C}$. The static magnetic field of $10 \mathrm{~T}$ was imposed on the whole samples during solidification. The obtained macrostructures are shown in Fig. 10. Because a coarse structure is observed in the sample 5, the imposition of the electrical current in initial stage of the solidification is crucial for refinement of the structure in this process. All of the region are refined in the samples 2, 3 and 4 while some area in the sample 1 is coarse. Therefore, the imposition of the electromagnetic vibration until solid fraction reaches 0.09 is not enough but until it reaches 0.44 is enough for the refinement of the whole region of the sample in this experimental condition.
Electrode
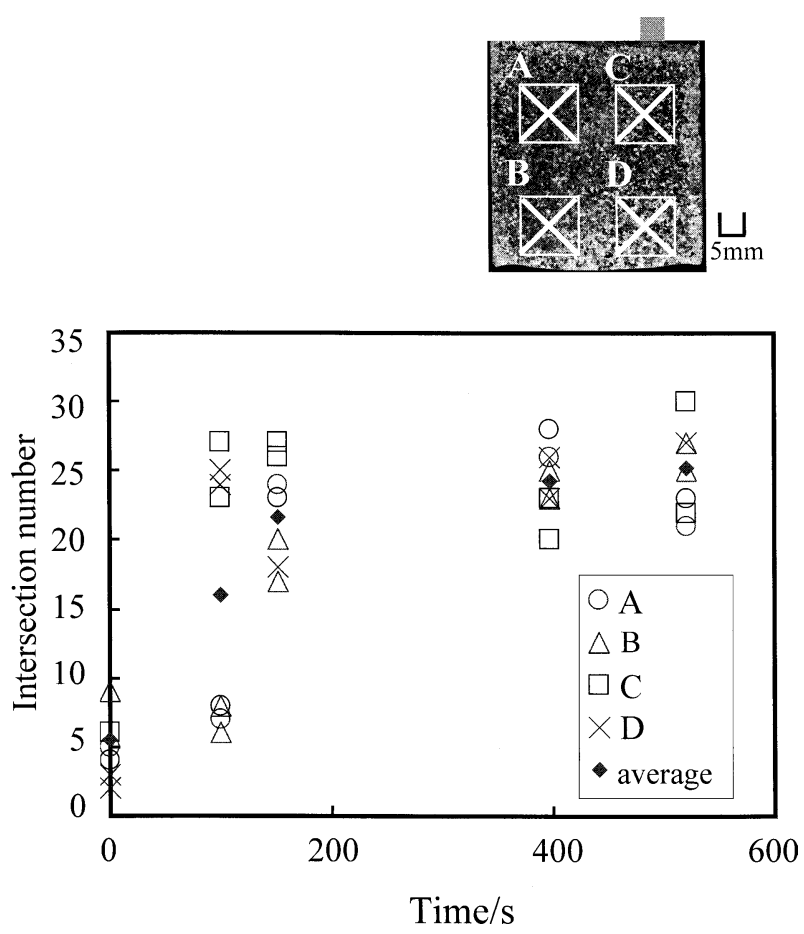

Fig. 11. Intersection number between grain boundary and diagonal of $10 \mathrm{~mm} \times 10 \mathrm{~mm}$ square as a function of imposing duration of electrical current.

The intersection number of grain boundaries with diagonals of a $10 \mathrm{~mm} \cdot 10 \mathrm{~mm}$ square for the samples $1,2,3,4$ and the sample without electrical current is shown in Fig. 11 as the function of the imposing time of the electrical current. The intersection numbers at the positions $\mathrm{C}$ and $\mathrm{D}$ drastically increase in the initial stage of the solidification while those at the positions A and B gradually increase, and the numbers at the all positions finally become almost the same value.

\section{Conclusion}

Effect of the operating parameter on the solidified structure of the $\mathrm{Sn}-\mathrm{Pb}$ alloy in the electromagnetic refining process of the solidified structure in which a static magnetic field and an alternating current were locally imposed on a sample during solidification have been experimentally investigated.

The followings are main results obtained in this research.

- Frequency of the electrical current dose not affect on the solidified structure.

- Threshold intensity of the electrical current for the refining of the solidified structure exists.

- Refining effect is mainly observed around the electrode in the case that the magnitude of the electrical current is around the threshold value.

- Imposition of the electromagnetic force in the initial stage of the solidification is crucial to obtain the refined structure in this process and the imposition in the middle stage has no refining function. 


\section{Acknowledgement}

This research was partially supported by the Ministry of Education, Culture, Sports, Science and Technology, Grantin-Aid for Scientific Research (B), (No.15360400), and Iron and Steel Institute of Japan.

\section{Nomenclature}

$B_{\mathrm{DC}}$ : Static magnetic flux density $(\mathrm{T})$

$c$ : Propagation velocity of ordinary compression waves $(\mathrm{m} / \mathrm{s})$

$J_{\mathrm{AC}}$ : Effective value of imposed alternating current density $\left(\mathrm{A} / \mathrm{m}^{2}\right)$

$k$ : Wave number $(1 / \mathrm{m})$

$l$ : Length of a liquid metal (m)

$N$ : Constant (-)

$P_{\mathrm{m}}:$ Constant $(\mathrm{Pa})$

$V_{\mathrm{m}}:$ Constant $(\mathrm{m} / \mathrm{s})$

$v_{x}$ : Velocity in $x$-direction $(\mathrm{m} / \mathrm{s})$

$x$ : Distance in $x$-direction $(\mathrm{m})$ $\gamma:$ Propagation velocity ratio $(-)$

$\delta$ : Electromagnetic skin layer $(\mathrm{m})$

$\rho:$ Density $\left(\mathrm{kg} / \mathrm{m}^{3}\right)$

$\sigma: \quad$ Electrical conductivity $(\mathrm{S} / \mathrm{m})$

$\phi:$ Constant (rad)

$\omega$ : Angular frequency $(\mathrm{rad} / \mathrm{s})$

\section{REFERENCES}

1) Y. Osawa, G. Arakane, S. Takamori, A. Sato and O. Ohashi: Chuuzoukougaku, 71 (1999), 98.

2) S. Amano, K. Iwai and S. Asai: ISIJ Int., 37 (1997), 962.

3) Q. Wang, T. Momiyama, K. Iwai and S. Asai: Mater. Trans. JIM, 41 (2000), 1742.

4) C. Vives: Metall. Mater. Trans. B, 27B (1996), 445.

5) A. Radjai, K. Miwa and T. Nishio: Metall. Mater. Trans. A, 29A (1998), 1477

6) A. Radjai and K. Miwa: Metall. Mater. Trans. A, 31A (2000), 755.

7) S. Kawai, Q. Wang, K. Iwai and S. Asai: Mater. Trans. JIM, 42 (2001), 275.

8) T. Takagi, K. Iwai and S. Asai: ISIJ Int., 43 (2003), 842. 